\title{
Idiopathic Pulmonary Lymphoid Diffuse Hyperplasia
}

\author{
P.A. Canessa1, L. Pratticò1, F. Fiasella1, A. Cavazza², \\ B. Bacigalupo 3 , F. Fedeli3
}

ABSTRACT: Idiopathic Pulmonary Lymphoid Diffuse Hyperplasia. P.A. Canessa, L. Pratticò, F. Fiasella, A. Cavazza, B. Bacigalupo, F. Fedeli.

We describe a 70 year old woman affected by diffuse pulmonary lymphoid hyperplasia (DPLH). DPLH is a rare clinical-pathological entity generally associated with connective tissue diseases, but we diagnosed our case as an idiopathic DPLH. To the best of our knowledge, this is the first case of idiopathic DPLH reported in medical literature.

Monaldi Arch Chest Dis 2009; 71: 2, 69-70.

1 UO Pneumologia Ospedale S Bartolomeo, Sarzana (SP).

2 UO Anatomia Patologica Arcispedale S Maria Nuova, Reggio Emilia.

3 UO Anatomia Patologica Ospedale S Andrea, La Spezia; Italy.

Correspondence: Pier Aldo Canessa, Ospedale S. Bartolomeo, via Cisa sud, 19038 Sarzana (SP), Italy; e-mail: pieraldo.canessa@asl5.liguria.it

\section{Case report}

In February 2003, a 70 year-old non-smoker housewife without any para-occupational condition was examined. She had a one-month's history of dyspnoea, cough and fever; antibiotics had been administrated for three weeks without improvement and she was admitted to the hospital for further evaluation.

She denied any drug use, a history of toxic inhalation or drug abuse. Physical examination was negative. The laboratory tests produced results that were within the normal range, and an ECG was normal.

Arterial blood gas values were: $\mathrm{pH} 7.461, \mathrm{PaO}_{2}$ $95.9 \mathrm{mmHg}, \mathrm{PaCO}_{2} 33.6 \mathrm{mmHg}$. A chest X-ray showed bilateral lower-zone coarse reticulonodular infiltrates with consolidated alveolar opacities.

The pulmonary function tests gave the following results: TLC $75 \%$ of predicted value, RV $76 \%$, VC $73 \%, \mathrm{FVC} 76 \%, \mathrm{FEV}_{1} 76 \%$, DLCO 53\%, DLCO/VA $56 \%$. The bronchodilatation test was negative.

A chest-CT showed a number of small nodular opacities in the centrilobular and in the subpleural areas of the superior and inferior lobes with interlobular septal thickening and with alveolar consolidation in inferior lobes (figure 1A).

The immunity tests were negative (Rheumatoid factor, ANA, anti SSA/Ro and anti SSB/La antibodies, anti Sm, anti RNP, SCL70, Jo1, ASMA, anticardiolipin antibodies).

No infectious pathogens were detected in blood (antibody dosage for toxoplasma, mycoplasma, mononucleosis, chlamydia, rubeola, herpes simplex, borrelia; CMV-DNA and CMV-antigen, HIV, Aspergillus), in urine (pneumococcus and legionella antigens) and in BAL (P Jirovecii, Koch Bacillus).
Bronchoscopy was normal; the transbronchial biopsies performed in the left lower lobe showed a dense lymphoid infiltration of the chorion. The BAL was: macrophages $29 \%$, neutrophils $28 \%$, lymphocytes $43 \%$ with CD4/CD8 ratio 0.4.

No specific diagnosis was reached, and a videothoracoscopic biopsy of the lingula was performed, showing a dense lymphoplasmacellular infiltration localised mostly along the lymphatic routes (fig. 2A). This infiltration had produced follicles and occasional lymphoepitelial complexes with small accumulations of fibrin, endoalveolar macrophages and foci of subacute organising pneumonia (figure 2B). A Grocott and a ZiehlNeelsen stains were negative. Immunohistochemically, the lymphoid infiltrate was mixed, B (CD20 +) and $\mathrm{T}(\mathrm{CD} 3+)$, and the plasmacells were polyclonal with light chain immunoglobulins. A bonemarrow biopsy was normal.

The patient was treated with $25 \mathrm{mg} /$ die of prednisone for six months, and she had a clinical improvement. In November 2003 chest CT was improvement (fig 1B), DLCO values were normal $(90 \%)$ and prednisone was reduced at $12.5 \mathrm{mg} / \mathrm{die}$. After 18 months autoimmunity tests remained negative. After 36 months the patient's follow up showed clinical and radiological stability with $12.5 \mathrm{mg} / \mathrm{die}$ of prednisone. She did not have any CVD clinical and serological evidence. This was the minimal effective dosage to control the disease; below this dosage, the patient suffered from coughing and dyspnoea.

\section{Discussion}

Lymphoid tissue is uncommon in normal adult human lungs. In non-smoking adults, the majority of 


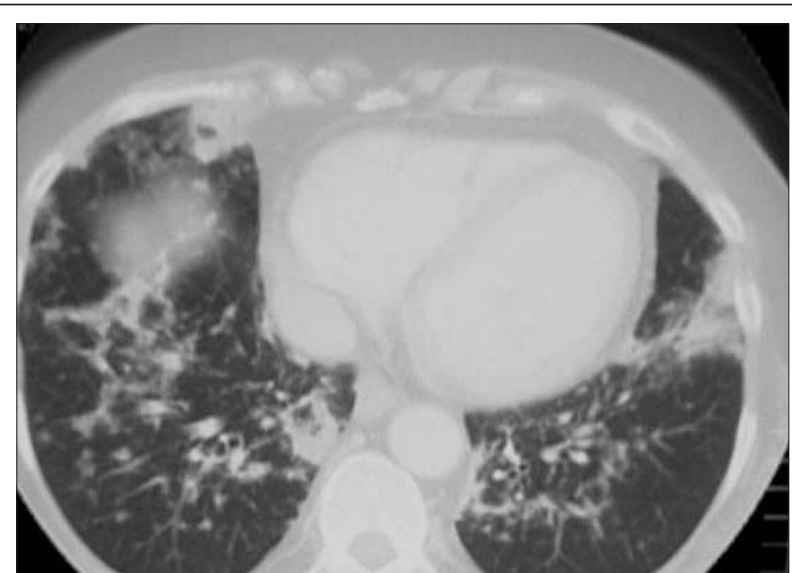

Fig. 1A. - Chest CT (February 2003) bilateral multiple nodules and alveolar consolidation areas.

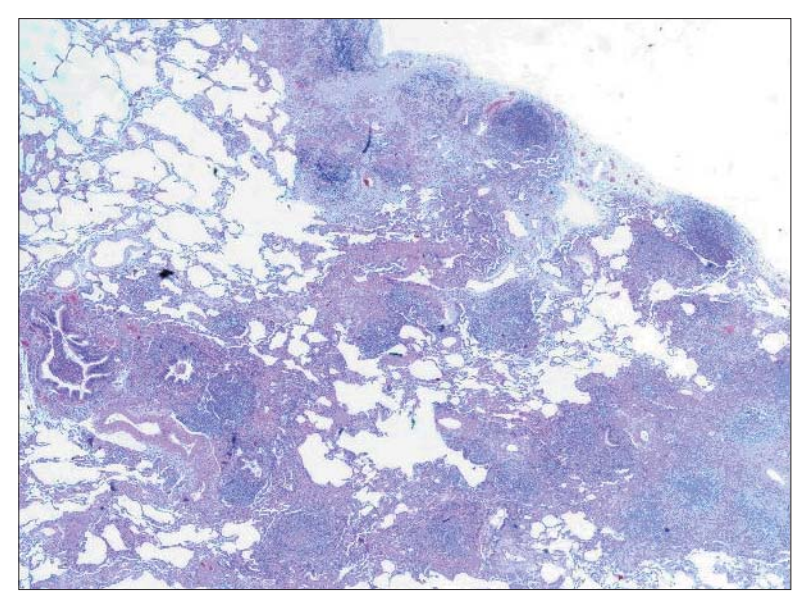

Fig. 2A. - Videothoracoscopic biopsy, showing lymphoid aggregates along the lymphatic routes (Hematoxylin-Eosin, 20x).

pulmonary lymphoid tissue is formed into poorly-organised aggregates predominantly located at bronchial divisions and adjacent to distal respiratory bronchioles [1]. Additionally, small numbers of lymphoid cells are occasionally found scattered beneath and between bronchial epithelial cells [2]. Approximately $60 \%$ of lung lymphoid cells are B cells, and the rest are $\mathrm{T}$ lymphocytes [3]. The constellation of non capsulated lymphoid follicles, cellular collections, and loosely distributed mucosal lymphocytes constitute the bronchus-associated lymphoid tissue (BALT) or "pulmonary microtonsils" [4].

The acquired benign diffuse proliferations of BALT includes follicular bronchitis/bronchiolitis (FBB); diffuse pulmonary lymphoid hyperplasia (DPLH); and lymphocitic interstitial pneumonia (LIP) [4].

DPLH is characterised by lymphoid infiltrates localised in the pleura, in the interlobular septae and along bronchovascular bundles, and differs from LIP because a significant alveolar septal involvement is lacking; however, a morphological overlap exists between DPLH, FBB and LIP [5]. Moreover, the differential diagnosis with lowgrade MALT lymphoma can be difficult and requires immunohistochemical tests.

DPLH is a rare clinical-pathological entity generally associated with connective tissue dis-

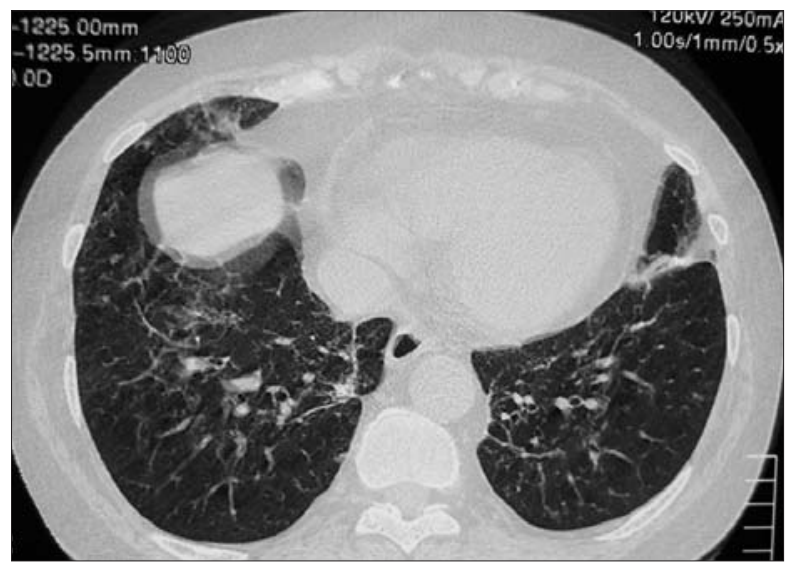

Fig. 1B. - Chest CT (November 2003) no nodules and reduction of alveolar consolidation.

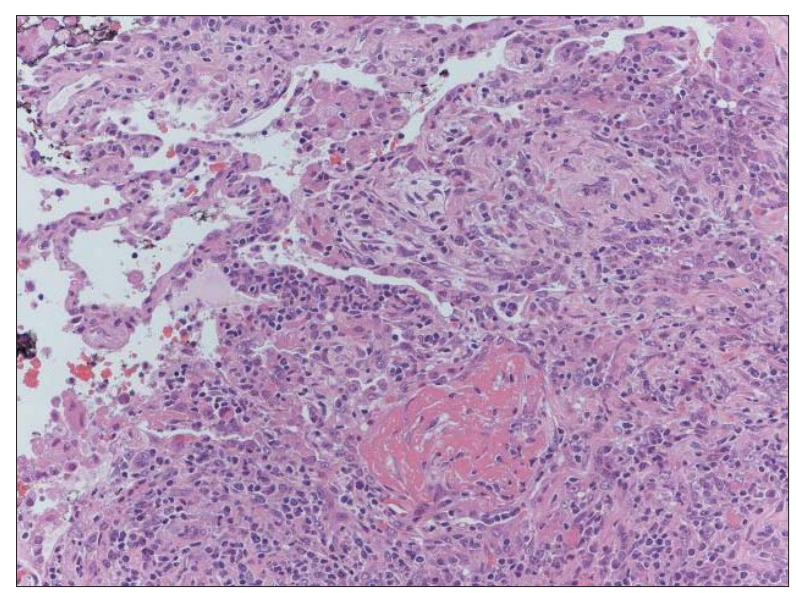

Fig. 2B. - Tiny foci of organising pneumonia with fibrin and intraalveolar macrophages were present. (Hematoxylin-Eosin, 200X)

eases, especially Sjögren's syndrome or rheumatoid arthritis [5], immunodeficiency syndrome [4] and infections [4].

In our patient's case, her history and laboratory tests have excluded any association between her condition and the diseases listed above. Therefore, we have diagnosed our case as an idiopathic DPLH. To the best of our knowledge, this is the first example of idiopathic DPLH reported in medical literature.

\section{References}

1. Koss M. Pulmonary lymphoid disorders. Semin Diagn Pathol 1995; 12: 158-171.

2. Pabst R, Gehrke I. Is the bronchus-associated lymphoid tissue (BALT) an integral structure of the lung in normal mammals, including humans? Am J Respir Cell Mol Biol 1990; 3: 131-135.

3. Sminia T, van der Brugge-Gamelkoorn G, Jeurissen S. Structure and function of bronchus-associated lymphoid tissue (BALT). Crit Rev Immunol 1989; 9: 119-150.

4. Swigris JJ, Berry GJ, Raffin TA, Kuschner WG: Lymphoid interstitial pneumonia. Chest 2002; 122: 21502164.

5. Nicholson AG, Colby TV, Wells AU. Histopathological approach to pattern of interstitial pneumonia in patients with connective tissue disorders. Sarcoidosis Vasc Diffuse Lung Dis 2002; 19: 10-17. 